\title{
Comment on Chen et al.: Patellar resurfacing versus nonresurfacing in total knee arthroplasty: a meta-analysis of randomised controlled trials
}

\author{
Chenli Ye $\cdot$ Guoping Liao $\cdot$ Shuai He $\cdot$ Zhongyi Zhang
}

Received: 14 May 2013 / Accepted: 15 May 2013 / Published online: 18 June 2013

(C) Springer-Verlag Berlin Heidelberg 2013

Dear Editor,

Recently, we read with great interest the article by Chen et al. [1] entitled "Patellar resurfacing versus nonresurfacing in total knee arthroplasty: a meta-analysis of randomised controlled trials" published in March 2013 in International Orthopaedics. Chen et al. performed a meta-analysis to make an estimation of patellar resurfacing versus non-resurfacing in total knee arthroplasty. It is an interesting study. Nevertheless, we have several queries which we would like to communicate to the authors.

1. Three electronic databases (MEDLINE, Embase and the Cochrane database) were systematically searched by the authors for randomised trials. However, the authors did not focus specifically or in any detail on the issue of the completeness of the search strategy report for databases. The search strategy report plays an important role in systematic reviews.

2. Publication language was limited to English in the metaanalysis. Therefore, the authors should mention the potential importance of language bias in the limitations of their meta-analysis.

3. Unpublished data (grey literature) were not included in the meta-analysis. Additionally, it is not sufficient that publication bias was only assessed by visual examination of the funnel plot. Funnel plot symmetry should be further assessed by statistical tests (e.g. Egger's linear regression test or Begg's rank correlation test). Moreover, in the meta-analysis, publication bias was only

C. Ye $\cdot \mathrm{S} . \mathrm{He} \cdot \mathrm{Z}$. Zhang $(\bowtie)$

Department of Pharmacy, Zhujiang Hospital, Southern Medical University, Guangzhou 510282, People's Republic of China

e-mail: zhang43499@sohu.com

G. Liao

Changning Hospital of Traditional Chinese Medicine, Changning 421500 Hunan Province, People's Republic of China assessed for reoperation following patellar resurfacing. Actually, publication bias should be assessed for other comparisons (such as post-operative anterior knee pain). Therefore, publication bias may be present, distorting the meta-analysis.

4. There are different approaches to patellar resurfacing in total knee arthroplasty, which would bring different results. If possible, we suggest that a meta-analysis of different operative treatments versus non-resurfacing treatment in total knee arthroplasty could be conducted.

5. It is not appropriate that summary risk ratio (RR) and risk difference (RD) estimates with corresponding $95 \%$ confidence intervals (CIs) were derived by using the method of Mantel-Haenszel (M-H) or inverse variance (IV) with the assumptions of a random effects model. However, studies should be combined by using the DerSimonian and Laird random effects model, which considers both within- and between-study variations [2].

We agree with the following conclusions of the authors: patellar resurfacing reduces the risk of reoperation after total knee arthroplasty, patellar resurfacing patients may make a difference in long-term follow-up (five or more years) of Knee Society scores, and more carefully and scientifically designed randomised controlled trials (RCTs) with large samples and long-term follow-up are required to further prove the claim.

\section{References}

1. Chen K, Li G, Fu D, Yuan C, Zhang Q, Cai Z (2013) Patellar resurfacing versus nonresurfacing in total knee arthroplasty: a meta-analysis of randomised controlled trials. Int Orthop 37:10751083

2. DerSimonian R, Laird N (1986) Meta-analysis in clinical trials. Control Clin Trials 7:177-188 